\title{
Quantization of soluble classical constrained systems
}

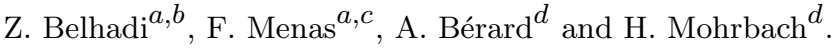 \\ $a$ - Laboratoire de physique et chimie quantique, Faculté des sciences. \\ Université Mouloud Mammeri, BP 17, 15000 Tizi Ouzou, Algérie. \\ $b$ - Laboratoire de physique théorique, Faculté des sciences exactes. \\ Université de Bejaia. 06000 Bejaia, Algérie. \\ $c$ - Ecole Nationale Préparatoire aux Etudes d'ingéniorat. Laboratoire de physique. \\ RN 5 Rouiba, Alger. Algérie. \\ $d$ - Equipe BioPhysStat, Laboratoire LCP-A2MC, ICPMB, IF CNRS N² 2843 \\ Université de Lorraine. 1 Bd Arago, 57078 Metz Cedex, France.
}

September 25, 2018

\begin{abstract}
The derivation of the brackets among coordinates and momenta for classical constrained systems is a necessary step toward their quantization. Here we present a new approach for the determination of the classical brackets which does neither require Dirac's formalism nor the symplectic method of Faddeev and Jackiw. This approach is based on the computation of the brackets between the constants of integration of the exact solutions of the equations of motion. From them all brackets of the dynamical variables of the system can be deduced in a straightforward way.
\end{abstract}

\section{Introduction}

The Hamiltonian method for the quantization of a dynamical system requires to postulate canonical Poisson brackets among coordinates and momenta. Then the correspondence principle is applied to determine the different commutators of the quantum operators associated to the classical dynamical variables. However, for singular Lagrangians or constrained systems, this procedure does not work and the brackets have to be determined and not postulated. Dirac has developed a general formalism for treating such systems 1, 2]. Dirac formalism has been widely used but the determination of the Hamiltonian and the Dirac brackets replacing the Poisson brackets can be very cumbersome. More recently Faddeev and Jackiw have proposed [3, 4] an alternative approach (FJ approach) based on the symplectic formalism and Darboux theorem (which is equivalent to an approach proposed by Souriau [5] as remarked in [6] and [7 ) to find the brackets and the Hamiltonian which can often avoid many of the steps of Dirac method.

Here we propose another approach for the determination of the Hamiltonian and the brackets for unconstrained and constrained systems which avoids the Dirac categorization of constraints and the Darboux theorem of the FJ approach. Our method is based on the computation of the brackets between the constants of integration of the solutions of the Euler-Lagrange equations of motion. 
From them all brackets among coordinates and momenta can be directly deduced. Therefore when a system is classically soluble, i.e the analytical solution of its equations of motion are known, it is possible to quantize the system without using Dirac or FJ formalisms.

In the two next sections, the general formalism for the computation of the brackets among the constants of integration and the link with Dirac and FJ methods is presented. Then in the following sections we provide several examples which are treated with the three methods. At the end of the paper we mention possible applications to field theory.

\section{Remark on the integration constants}

Consider a system of $N$ degrees of freedom characterized by its generalized coordinates $q=$ $\left(q_{1}, q_{2}, \ldots, q_{N}\right)$ and conjugate momenta $p=\left(p_{1}, p_{2}, \ldots, p_{N}\right)$. Now let $f=f(q, p)$ and $g=g(q, p)$ be two arbitrary functions. We define an antisymmetric bilinear bracket satisfying the Leibniz rule and the Jacobi identities, such that:

$$
\{f, g\}=\sum_{i, j=1}^{N}\left(\left\{q_{i}, q_{j}\right\} \frac{\partial f}{\partial q_{i}} \frac{\partial g}{\partial q_{j}}+\left\{p_{i}, p_{j}\right\} \frac{\partial f}{\partial p_{i}} \frac{\partial g}{\partial p_{j}}+\left\{q_{i}, p_{j}\right\}\left(\frac{\partial f}{\partial q_{i}} \frac{\partial g}{\partial p_{j}}-\frac{\partial f}{\partial p_{j}} \frac{\partial g}{\partial q_{i}}\right)\right)
$$

At this level this bracket is not yet specified. It can be either the Poisson, or Dirac or also a FJ bracket. Now, suppose that $q$ and $p$ are functions of time and of new variables $R=\left(R_{1}, R_{2}, \ldots, R_{M}\right)$ with $M \leq 2 N$, i.e $11=q(R, t)$ and $p=p(R, t)$. Then Eq. (11) becomes

$$
\begin{aligned}
\{f, g\}= & \sum_{i, j=1}^{N} \sum_{k, l=1}^{M}\left(\left\{q_{i}, q_{j}\right\} \frac{\partial f}{\partial R_{k}} \frac{\partial R_{k}}{\partial q_{i}} \frac{\partial g}{\partial R_{l}} \frac{\partial R_{l}}{\partial q_{j}}+\left\{p_{i}, p_{j}\right\} \frac{\partial f}{\partial R_{k}} \frac{\partial R_{k}}{\partial p_{i}} \frac{\partial g}{\partial R_{l}} \frac{\partial R_{l}}{\partial p_{j}}\right) \\
& +\sum_{i, j=1}^{N} \sum_{k, l=1}^{M}\left\{q_{i}, p_{j}\right\}\left(\frac{\partial f}{\partial R_{k}} \frac{\partial R_{k}}{\partial q_{i}} \frac{\partial g}{\partial R_{l}} \frac{\partial R_{l}}{\partial p_{j}}-\frac{\partial f}{\partial R_{k}} \frac{\partial R_{k}}{\partial q_{j}} \frac{\partial g}{\partial R_{l}} \frac{\partial R_{l}}{\partial p_{i}}\right)
\end{aligned}
$$

which simplifies to

$$
\begin{aligned}
\{f, g\}= & \sum_{k, l=1}^{M}\left[\sum_{i, j=1}^{N}\left\{q_{i}, q_{j}\right\} \frac{\partial R_{k}}{\partial q_{i}} \frac{\partial R_{l}}{\partial q_{j}}+\sum_{i, j=1}^{N}\left\{p_{i}, p_{j}\right\} \frac{\partial R_{k}}{\partial p_{i}} \frac{\partial R_{l}}{\partial p_{j}}\right. \\
& \left.+\sum_{i, j=1}^{N}\left\{q_{i}, p_{j}\right\}\left(\frac{\partial R_{k}}{\partial q_{i}} \frac{\partial R_{l}}{\partial p_{j}}-\frac{\partial R_{k}}{\partial q_{j}} \frac{\partial R_{l}}{\partial p_{i}}\right)\right] \frac{\partial f}{\partial R_{k}} \frac{\partial g}{\partial R_{l}}
\end{aligned}
$$

and leads to the expression of $\{f, g\}$ in terms of $\left\{R_{k}, R_{l}\right\}$ :

$$
\{f, g\}=\sum_{k, l=1}^{M}\left\{R_{k}, R_{l}\right\} \frac{\partial f}{\partial R_{k}} \frac{\partial g}{\partial R_{l}}
$$

With the introduction of the new variables, the sum goes from 1 to $M(M \leq 2 N)$. Thus, the presence of constraints reduces the number of variables and we will only work with the free variables. Now,

\footnotetext{
${ }^{1} M=2 N$ corresponds to an unconstrained system. For the constrained systems, each constraint eliminates one variable.
} 
if $f=q_{i}$ or $p_{i}$ and $g=H$ the Hamiltonian of the system, we obtain the following equations (using Hamilton equations for $i=1$ to $N$ )

$$
\begin{aligned}
& \frac{d q_{i}}{d t}=\left\{q_{i}, H\right\} \quad \Rightarrow \quad \frac{\partial q_{i}}{\partial t}+\sum_{j=1}^{M} \frac{\partial q_{i}}{\partial R_{j}} \frac{d R_{j}}{d t}=\sum_{j, k=1}^{M}\left\{R_{j}, R_{k}\right\} \frac{\partial q_{i}}{\partial R_{j}} \frac{\partial H}{\partial R_{k}} \\
& \frac{d p_{i}}{d t}=\left\{p_{i}, H\right\} \quad \Rightarrow \quad \frac{\partial p_{i}}{\partial t}+\sum_{j=1}^{M} \frac{\partial p_{i}}{\partial R_{j}} \frac{d R_{j}}{d t}=\sum_{j, k=1}^{M}\left\{R_{j}, R_{k}\right\} \frac{\partial p_{i}}{\partial R_{j}} \frac{\partial H}{\partial R_{k}}
\end{aligned}
$$

Now suppose that $R_{k}, k=1 \ldots M$ are constants of motion (first integrals), denoted $R_{k}=C_{k}, k=$ $1 \ldots M$, then the above equations will be reduced to the form

$$
\begin{aligned}
& \frac{\partial q_{i}}{\partial t}=\sum_{j, k=1}^{M}\left\{C_{j}, C_{k}\right\} \frac{\partial q_{i}}{\partial C_{j}} \frac{\partial H}{\partial C_{k}} \\
& \frac{\partial p_{i}}{\partial t}=\sum_{j, k=1}^{M}\left\{C_{j}, C_{k}\right\} \frac{\partial p_{i}}{\partial C_{j}} \frac{\partial H}{\partial C_{k}} \quad i=1 \ldots N
\end{aligned}
$$

Therefore the dynamics of the system can be expressed in terms of the brackets among the integration constants, without ever specifying the nature of the bracket (Poisson, Dirac or FJ brackets ). Therefore the relations Eqs.(4) are universal and common to all types of brackets. With the introduction of the notation $\left.\xi_{i}\right|_{i=1, \ldots 2 N}=\left(q_{i}, p_{i}\right)$ we can now write Eqs.(44) in a compact form :

$$
\frac{\partial \xi_{i}}{\partial t}=\sum_{j, k=1}^{M}\left\{C_{j}, C_{k}\right\} \frac{\partial \xi_{i}}{\partial C_{j}} \frac{\partial H}{\partial C_{k}} \quad i=1 \ldots 2 N
$$

These relations can obtained in the same manner as we did by starting from the equations of motion

$$
\dot{\xi}_{i}=\left\{\xi_{i}, H\right\}=\sum_{j=1}^{2 N}\left\{\xi_{i}, \xi_{j}\right\} \frac{\partial H}{\partial \xi_{j}} \quad i=1 \ldots 2 N
$$

used in the FJ approach.

\section{Method of Integration constants: Constrained (and un- constrained) systems}

In this section we use the previous properties Eqs. (44) to determine the brackets of any exactly soluble system. At the end the canonical quantization allows us to build the quantum version. Although our approach (called in the rest of the paper the CI method) is applicable to systems without constraints, it is for constrained systems that it shows its main interest. Indeed, we propose a new method which greatly facilitates the computation of the Dirac brackets, while avoiding both the complicated Dirac algorithm and the Darboux theorem in the case of the FJ approach.

Consider a classical system described by a singular autonomous Lagrangian $L(q, \dot{q})$ where $q=$ $\left(q_{1}, \ldots, q_{N}\right)$ are the generalized coordinates and $\dot{q}=\left(\dot{q}_{1}, \ldots, \dot{q}_{N}\right)$ the generalized velocities. Suppose 
we know the (general) analytical solutions $q(t)=\tilde{q}(t, C)$ of the Euler-Lagrange equations and the momenta $p(t)=\tilde{p}(t, C)\left(p_{i}=\frac{\partial L}{\partial \dot{x}_{i}}\right)$, where $C=\left(C_{1}, C_{2}, \ldots, C_{M}\right)$ is the set of constants of integration. For constrained systems we have obviously $M<2 N$.

Before going further, it is necessary to distinguish between two cases: the first is when there are no arbitrary functions in the solutions (no gauge symmetry), the second is otherwise. In the later case with a gauge symmetry, we must first choose the arbitrary functions once by adding new conditions (fixing the gauge) before moving to the canonical formalism and defining any brackets.

From the analytical solutions of the equations of motion we can write the Hamiltonian 2 as $H(q(t), p(t))=H(\tilde{q}(t, C), \tilde{p}(t, C))$ and from Eqs. (3) we deduce the fundamental equations

$$
\begin{aligned}
\frac{\partial}{\partial t} \tilde{q}_{i}(t, C)=\sum_{j, k=1}^{M}\left\{C_{j}, C_{k}\right\} \frac{\partial \tilde{q}_{i}}{\partial C_{j}} \frac{\partial H}{\partial C_{k}} \quad i=1 \ldots N \\
\frac{\partial}{\partial t} \tilde{p}_{i}(t, C)=\sum_{j, k=1}^{M}\left\{C_{j}, C_{k}\right\} \frac{\partial \tilde{p}_{i}}{\partial C_{j}} \frac{\partial H}{\partial C_{k}} \quad i=1 \ldots N
\end{aligned}
$$

These $2 N$ equations contain $M(M-1) / 2$ unknown brackets $\left\{C_{j}, C_{k}\right\}$, with $j, k=1 \ldots M$. Our method consist in determining the brackets $\left\{C_{j}, C_{k}\right\}$ from the Eqs.(7) via a simple identification. But, with this procedure, we easily see that only the brackets containing at least one integration constant in the expression of the Hamiltonian are available. To solve this problem, we must add supplementary terms to the Lagrangian. In other words, if for example the bracket $\left\{C_{i}, C_{j}\right\}$ is not accessible and if one of these constants appears in the expression of the generalized coordinate $q_{i}$, one has to add a term of the form $\eta q_{i}$ to the Lagrangian, redo all the calculations and put $\eta=0$ at the end (as an illustration see example 4.2). Using the brackets $\left\{C_{i}, C_{j}\right\}$ we can compute the brackets $\left\{q_{i}, q_{j}\right\},\left\{p_{i}, p_{j}\right\}$ and $\left\{q_{i}, p_{j}\right\}$ more easily than with any other existing approaches. If the result of the calculation depends on the integration constants, it is possible to make them disappear by inverting the solutions $\tilde{q}(t, C)$ and $\tilde{p}(t, C)$. At this time, to be sure of the validity of our calculations, we can just verify that $\tilde{q}(t, C)$ and $\tilde{p}(t, C)$ are solutions of the equations of motion obtained from the Hamilton equations using these fundamental brackets. We see that in our method we do not even talk about constraints unlike other approaches.

These brackets are essential for the quantization of the system by introducing the quantum operators $\widehat{q}$ and $\widehat{p}$ and the principle of correspondence we have :

$$
[f(\hat{q}, \hat{p}), g(\hat{q}, \hat{p})]=i \hbar\{f(q, \widehat{p), g(}(q, p)\}
$$

Note, that it might look surprising to see brackets between constants and the derivative of functions with respect to those constants. Actually, these constants are first integrals and must be considered as in the Hamilton-Jacobi formalism which also treats the constants as variables.

\footnotetext{
${ }^{2} \mathrm{We}$ can also obtain the Hamiltonian by putting the solutions into the Legendre transformation $H=$ $\sum_{i} \frac{d \tilde{q}_{i}(t, C)}{d t} \tilde{p}_{i}(t, C)-L\left(\tilde{q}_{i}(t, C), \frac{d \tilde{q}_{i}(t, C)}{d t}\right)$. In this way we do not have to inverse the momenta with respect to the velocities.
} 


\section{Applications}

In this section we present several appealing examples. As a first application of the method we consider the isotonic oscillator which is an unconstrained system. This case shows that the method is general and thus valid for constrained and unconstrained systems. The second application deals with a singular autonomous Lagrangian which exemplifies the previous discussion about the procedure when $\left\{C_{i}, C_{j}\right\}$ is not directly accessible (when the Hamiltonian does not contain $C_{i}$ or $C_{j}$ ). The other examples concern different constrained systems which are studied with the Dirac, FJ and our method for comparison.

\subsection{Regular Lagrangian}

For the first example we consider an unconstrained system namely the Lagrangian of the isotonic (anharmonic) oscillator:

$$
L=\frac{1}{2} \dot{x}^{2}-\frac{1}{2} \omega^{2} x^{2}-\frac{k}{x^{2}}
$$

The Euler-Lagrange equations lead to the following non-linear equation

$$
\ddot{x}+\omega^{2} x-\frac{2 k}{x^{3}}=0
$$

whose solution is (see ref: [8]) $x(t)=\frac{1}{A \omega} \sqrt{\left(A^{4} \omega^{2}-2 k\right) \sin ^{2}(w t+\phi)+2 k}$ where $A$ and $\phi$ are the constants of integration. The conjugate momentum is given by $p_{x}(t)=\dot{x}(t)=\frac{\left(A^{4} \omega^{2}-2 k\right) \sin (w t+\phi) \cos (w t+\phi)}{A \sqrt{\left(A^{4} \omega^{2}-2 k\right) \sin ^{2}(w t+\phi)+2 k}}$. Expressing the Hamiltonian $H=\frac{1}{2} p_{x}^{2}+\frac{1}{2} \omega^{2} x^{2}+\frac{k}{x^{2}}$ in terms of these constants we obtain

$$
H=\frac{A^{4} \omega^{2}+2 k}{2 A^{2}}
$$

From the Hamilton equation $\dot{x}=\{x, H\}$ and the property Eq. (7) we obtain $\frac{\partial x}{\partial t}=\{\phi, A\} \frac{\partial x}{\partial \phi} \frac{\partial H}{\partial A}$ from which we obtain the bracket between the constants of integration

$$
\{\phi, A\}=\frac{A^{3} \omega}{A^{4} \omega^{2}-2 k}
$$

From Eq. (2) we obtain the brackets among the dynamical variables:

$$
\left\{x, p_{x}\right\}=\{\phi, A\}\left(-\frac{\partial x}{\partial A} \frac{\partial p_{x}}{\partial \phi}+\frac{\partial x}{\partial \phi} \frac{\partial p_{x}}{\partial A}\right)
$$

A short computation shows that $-\frac{\partial x}{\partial A} \frac{\partial p_{x}}{\partial \phi}+\frac{\partial x}{\partial \phi} \frac{\partial p_{x}}{\partial A}=\frac{A^{4} \omega^{2}-2 k}{A^{3} \omega}$ so that we retrieve the canonical Poisson bracket

$$
\left\{x, p_{x}\right\}=1
$$

as expected for an unconstrained system. 


\subsection{Singular autonomous Lagrangians: first example}

Consider a constrained system described by the Lagrangian :

$$
L=\frac{\dot{x}^{2}}{2}+x \dot{y}-y \dot{z}
$$

This system gives an illustration of the discussed procedure when the Hamiltonian does not contain all constants of integration. The Euler-Lagrange equations are

$$
\begin{array}{r}
\ddot{x}-\dot{y}=0 \\
\dot{x}+\dot{z}=0 \\
\dot{y}=0
\end{array}
$$

whose analytical solutions are :

$$
x(t)=a t+b \quad y(t)=c \quad z(t)=-a t+d
$$

where $a, b, c$ and $d$ are the constants of integration. From the conjugate momenta $p_{x}=\dot{x}, p_{y}=x$ and $p_{z}=-y$, and the Legendre transformation we obtain the Hamiltonian

$$
H=\frac{p_{x}^{2}}{2}=\frac{a^{2}}{2}
$$

From this Hamiltonian only the brackets containing $a$ are accessible. To solve this issue we introduce a new Lagrangian

$$
L=\frac{\dot{x}^{2}}{2}+x \dot{y}-y \dot{z}-\lambda x-\xi y
$$

where $\lambda$ and $\xi$ are real parameters. The new equations of motion are thus $\ddot{x}-\dot{y}+\lambda=0, \dot{x}+\dot{z}+\xi=0$ and $\dot{y}=0$. The general solution of this system reads

$$
x(t)=-\frac{\lambda}{2} t^{2}+a t+b \quad y(t)=c \quad z(t)=\frac{\lambda}{2} t^{2}-a t-\xi t+d
$$

The Hamiltonian now reads $H=\frac{p_{x}^{2}}{2}+\lambda x+\xi y$ or in terms of the constants of integration

$$
H=\frac{1}{2} a^{2}+\lambda b+\xi c
$$

From the Hamilton equations we can directly obtain the brackets between the different constants of integration. Indeed we have

$$
\dot{x}=\{x, H\} \Rightarrow-\lambda t+a=t \lambda\{a, b\}+\xi t\{a, c\}+a\{b, a\}+\xi\{b, c\}
$$

and

$$
\dot{z}=\{z, H\} \Rightarrow \lambda t-a-\xi=-t \lambda\{a, b\}-\xi t\{a, c\}+a\{d, a\}+\lambda t\{d, b\}+\xi\{d, c\}
$$

which by identification gives:

$$
\begin{aligned}
& \{a, b\}=\{a, d\}=-1 \\
& \{c, d\}=1 \\
& \{a, c\}=\{b, c\}=\{b, d\}=0
\end{aligned}
$$


A direct calculation using these results and the solution Eq. (9) leads the following brackets among the dynamical variables

$$
\begin{aligned}
\left\{x, p_{x}\right\} & =\{y, z\}=\left\{z, p_{z}\right\}=1 \\
\left\{p_{x}, p_{y}\right\} & =\left\{p_{x}, z\right\}=-1
\end{aligned}
$$

These brackets do not depend on the parameters $\lambda$ and $\xi$ and are thus unchanged if we put $\lambda=\xi=0$. In other words they are also the brackets of the initial Lagrangian $L=\frac{\dot{x}^{2}}{2}+x \dot{y}-y \dot{z}-\lambda x-\left.\xi y\right|_{\lambda=\xi=0}=$ $\frac{\dot{x}^{2}}{2}+x \dot{y}-y \dot{z}$.

\subsection{Singular autonomous Lagrangians: second example}

Consider a system described by the Lagrangian :

$$
L=\frac{\dot{x}^{2}}{2}+\frac{x^{2}}{2} \dot{y}-\frac{x^{2}}{2} y
$$

\subsubsection{CI method}

The Euler-Lagrange equations are

$$
\begin{array}{rlrl}
\ddot{x} & =x \dot{y}-x y & x \dot{x}=\frac{x^{2}}{2} \\
p_{x}=\dot{x} & p_{y}=\frac{x^{2}}{2}
\end{array}
$$

whose analytical solutions are :

$$
\begin{array}{rlrl}
x(t) & =a e^{-\frac{1}{2} t} & y(t) & =b e^{t}-\frac{1}{4} \\
p_{x}(t) & =-\frac{a}{2} e^{-\frac{1}{2} t}=-\frac{x}{2} & p_{y}(t) & =\frac{a^{2}}{2} e^{-t}
\end{array}
$$

where $a$ and $b$ are the constants of integration. Expressing the Hamiltonian in terms of these constants we have

$$
H=\left(p_{x}^{2}+x^{2} y\right) / 2=a^{2} b / 2
$$

From Eqs.(77) we have for the variable $x$,

$$
\dot{x}=\{x, H\} \quad \Rightarrow \quad \frac{a}{2} e^{-\frac{1}{2} t}=-\left\{a e^{-\frac{1}{2} t}, \frac{a^{2} b}{2}\right\} \quad \Rightarrow \quad \frac{a}{2} e^{-\frac{1}{2} t}=-e^{-\frac{1}{2} t} \frac{a^{2}}{2}\{a, b\}
$$

and then by identification

$$
\{a, b\}=\frac{-1}{a}
$$

From this brackets we obtain directly all other brackets among the dynamical variables. For instance:

$$
\{x, y\}=\left\{a e^{-\frac{1}{2} t}, b e^{t}-\frac{1}{4}\right\}=e^{\frac{1}{2} t}\{a, b\}=\frac{-1}{a} e^{\frac{1}{2} t}=-\frac{1}{x} .
$$


The other brackets are calculated in the same way, leading to

$$
\begin{aligned}
& \left\{x, p_{x}\right\}=\left\{x, p_{y}\right\}=\left\{p_{x}, p_{y}\right\}=0 \\
& \left\{y, p_{x}\right\}=-\frac{1}{2 x} \\
& \left\{y, p_{y}\right\}=1
\end{aligned}
$$

To check the validity of the results we determine the Hamilton equations by using the derived brackets, which are:

$$
\begin{aligned}
\dot{x} & =\{x, H\} \Rightarrow \dot{x}=-x / 2 ; \dot{y}=\{y, H\} \Rightarrow \dot{y}=-p_{x} /(2 x)+y \\
\dot{p}_{x} & =\left\{p_{x}, H\right\} \Rightarrow \dot{p}_{x}=x / 4 ; \dot{p}_{y}=\left\{p_{y}, H\right\} \Rightarrow \dot{p}_{y}=-x^{2} / 2
\end{aligned}
$$

These equations are equivalent to the Euler-Lagrange equations, which validates our approach for this first example.

\subsubsection{Dirac approach}

For comparison we consider the same Lagrangian with the Dirac treatment. First we deduce the momenta $p_{x}=\dot{x}$ and $p_{y}=\frac{x^{2}}{2}$ and the canonical Hamiltonian

$$
H_{c}=\frac{p_{x}^{2}}{2}+\frac{x^{2}}{2} y
$$

As we have a primary constraint $\phi=p_{y}-\frac{x^{2}}{2}=0$, we introduce the total Hamiltonian $H_{T}=H_{c}+\lambda \phi$ where $\lambda$

is a Lagrange multiplier. The consistency condition $\dot{\phi} \approx\left\{\phi, H_{T}\right\}_{P B} \approx\left\{\phi, H_{c}\right\}_{P B} \approx 0$ (where $\{,\}_{P B}$ denotes the Poisson bracket) leads to a secondary constraint $\zeta=\frac{x}{2}+p_{x} \approx 0$. The associated consistency condition $\dot{\zeta} \approx\left\{\zeta, H_{c}\right\}_{P B}+\lambda\{\zeta, \phi\}_{P B} \approx 0$ leads us to the following expression $\lambda \approx y-\frac{p_{x}}{2 x}$.

As $\{\phi, \zeta\}_{P B} \approx-x \neq 0$, the constraints are second class and the matrix of constraints is given by

$$
M=\left(\begin{array}{ll}
\{\phi, \phi\}_{P B} & \{\phi, \zeta\}_{P B} \\
\{\zeta, \phi\}_{P B} & \{\zeta, \zeta\}_{P B}
\end{array}\right)=\left(\begin{array}{ll}
0 & -x \\
x & 0
\end{array}\right)
$$

From the different elements of the inverse matrix

$$
M^{-1}=\left(\begin{array}{ll}
\{\phi, \phi\}_{P B}^{-1} & \{\phi, \zeta\}_{P B}^{-1} \\
\{\zeta, \phi\}_{P B}^{-1} & \{\zeta, \zeta\}_{P B}^{-1}
\end{array}\right)=\left(\begin{array}{ll}
0 & \frac{1}{x} \\
-\frac{1}{x} & 0
\end{array}\right)
$$

and those of the matrix of constraints, we can obtain the Dirac bracket $\{f, g\}_{D}$ among arbitrary functions which is defined as

$$
\{f, g\}_{D}=\{f, g\}_{P B}-\left\{f, \phi_{1}\right\}_{P B}\left\{\phi_{1}, \phi_{2}\right\}_{P B}^{-1}\left\{\phi_{2}, g\right\}_{P B}-\left\{f, \phi_{2}\right\}_{P B}\left\{\phi_{2}, \phi_{1}\right\}_{P B}^{-1}\left\{\phi_{1}, g\right\}_{P B}
$$

Applying this formula to the coordinates and momenta we get the following brackets: 


$$
\begin{aligned}
\{x, y\}_{D} & =\{x, y\}_{P B}-\{x, \phi\}_{P B} \frac{1}{x}\{\zeta, y\}_{P B}+\{x, \zeta\}_{P B} \frac{1}{x}\{\phi, y\}_{P B}=-\frac{1}{x} \\
\left\{x, p_{x}\right\}_{D} & =\left\{x, p_{x}\right\}_{P B}-\{x, \phi\}_{P B} \frac{1}{x}\left\{\zeta, p_{x}\right\}_{P B}+\{x, \zeta\}_{P B} \frac{1}{x}\left\{\phi, p_{x}\right\}_{P B}=0 \\
\left\{x, p_{y}\right\}_{D} & =\left\{x, p_{y}\right\}_{P B}-\{x, \phi\}_{P B} \frac{1}{x}\left\{\zeta, p_{y}\right\}_{P B}+\{x, \zeta\}_{P B} \frac{1}{x}\left\{\phi, p_{y}\right\}_{P B}=0 \\
\left\{y, p_{x}\right\}_{D} & =\left\{y, p_{x}\right\}_{P B}-\{y, \phi\}_{P B} \frac{1}{x}\left\{\zeta, p_{x}\right\}_{P B}+\{y, \zeta\}_{P B} \frac{1}{x}\left\{\phi, p_{x}\right\}_{P B}=-\frac{1}{2 x} \\
\left\{y, p_{y}\right\}_{D} & =\left\{y, p_{y}\right\}_{P B}-\{y, \phi\}_{P B} \frac{1}{x}\left\{\zeta, p_{y}\right\}_{P B}+\{y, \zeta\}_{P B} \frac{1}{x}\left\{\phi, p_{y}\right\}_{P B}=1 \\
\left\{p_{x}, p_{y}\right\}_{D} & =\left\{p_{x}, p_{y}\right\}_{P B}-\left\{p_{x}, \phi\right\}_{P B} \frac{1}{x}\left\{\zeta, p_{y}\right\}_{P B}+\left\{p_{x}, \zeta\right\}_{P B} \frac{1}{x}\left\{\phi, p_{y}\right\}_{P B}=0
\end{aligned}
$$

which are the same as with the CI method.

\subsubsection{Faddeev-Jackiw approach}

It is instructive to compare our method to the FJ approach. The initial Lagrangian is written in the following form, that we call the FJ Lagrangian

$$
L_{F J}=\dot{x} p_{x}+\dot{y} p_{y}-H=z \dot{x}+\frac{x^{2}}{2} \dot{y}-\frac{z^{2}}{2}-\frac{x^{2}}{2} y
$$

using the primary constraint $p_{y}=\frac{x^{2}}{2}$ and the notation $z=p_{x}$. In this approach one has to treat $x$, $y$ and $z$ as independent variables. Then the Euler-Lagrange equations 3 are

$$
\begin{aligned}
-\dot{z} & =-x \dot{y}+x y \\
-x \dot{x} & =+\frac{x^{2}}{2} \\
0 & =-\dot{x}+z
\end{aligned}
$$

which can be written in matrix form:

$$
\underbrace{\left(\begin{array}{ccc}
0 & x & -1 \\
-x & 0 & 0 \\
1 & 0 & 0
\end{array}\right)}_{f}\left(\begin{array}{l}
\dot{x} \\
\dot{y} \\
\dot{z}
\end{array}\right)=\left(\begin{array}{c}
x y \\
\frac{x^{2}}{2} \\
z
\end{array}\right)
$$

The matrix $f$ is singular and antisymmetric $(\operatorname{det}(f)=0)$. The only zero mode is ( $\left.\begin{array}{lll}0 & 1 & x\end{array}\right)$, and we obtain the secondary constraint by multiplying the last equation by this zero mode

$$
\left(\begin{array}{lll}
0 & 1 & x
\end{array}\right)\left(\begin{array}{c}
x y \\
\frac{x^{2}}{2} \\
z
\end{array}\right)=0
$$

\footnotetext{
${ }^{3}$ It is useful to write the Euler-Lagrange's equations under the form $-\frac{d}{d t} \frac{\partial L}{\partial \dot{q}_{i}}=-\frac{\partial L}{\partial q_{i}}$ in order to have directly the matrix $f$ antisymmetric.
} 
from which we obtain the constraint $\frac{x}{2}+z=0$, which is the same secondary constraint as with Dirac approach. This constraint must be conserved in time $\left(\frac{\dot{x}}{2}+\dot{z}=0\right)$. To assure that we define a new Lagrangian by adding the term $\lambda\left(\frac{\dot{x}}{2}+\dot{z}\right)$ to the Lagrangian $L_{F J}$. This term can be transformed in $-\dot{\lambda}\left(\frac{x}{2}+z\right)$ to give the following expression

$$
L_{F J}^{1}=z \dot{x}+\frac{x^{2}}{2} \dot{y}-\frac{z^{2}}{2}-\frac{x^{2}}{2} y+\dot{\lambda}\left(\frac{x}{2}+z\right)
$$

where $\lambda$ is a Lagrange multiplier which is considered as a new variable in the following. Therefore the new Euler-Lagrange equations are

$$
\begin{aligned}
-\dot{z} & =-x \dot{y}+x y-(1 / 2) \dot{\lambda} \\
-x \dot{x} & =\frac{x^{2}}{2} \\
0 & =-\dot{x}+z-\dot{\lambda} \\
-\left(\frac{\dot{x}}{2}+\dot{z}\right) & =0
\end{aligned}
$$

which can be written in matrix form as:

$$
\underbrace{\left(\begin{array}{cccc}
0 & x & -1 & +1 / 2 \\
-x & 0 & 0 & 0 \\
1 & 0 & 0 & 1 \\
-1 / 2 & 0 & -1 & 0
\end{array}\right)}_{f}\left(\begin{array}{c}
\dot{x} \\
\dot{y} \\
\dot{z} \\
\dot{\lambda}
\end{array}\right)=\begin{aligned}
& x y \\
& \frac{x^{2}}{2} \\
& z \\
& 0
\end{aligned}
$$

where $f$ is now antisymmetric and invertible as $\operatorname{det}(f)=x^{2} \neq 0$. The inverse of this matrix is :

$$
f^{-1}=\left(\begin{array}{cccc}
\{x, x\} & \{x, y\} & \{x, z\} & \{x, \lambda\} \\
\{y, x\} & \{y, y\} & \{y, z\} & \{y, \lambda\} \\
\{z, x\} & \{z, y\} & \{z, z\} & \{z, \lambda\} \\
\{\lambda, x\} & \{\lambda, y\} & \{\lambda, z\} & \{\lambda, \lambda\}
\end{array}\right)=\left(\begin{array}{cccc}
0 & -\frac{1}{x} & 0 & 0 \\
\frac{1}{x} & 0 & -\frac{1}{2 x} & -\frac{1}{x} \\
0 & \frac{1}{2 x} & 0 & -1 \\
0 & \frac{1}{x} & 1 & 0
\end{array}\right)
$$

Then it follows that

$$
\{x, y\}=-1 / x \quad ; \quad\{y, z\}=-\frac{1}{2 x} ; \quad\{x, z\}=0
$$

To have the brackets with $p_{y}$ we use the primary constraint $p_{y}=\frac{x^{2}}{2}$. We have obtained the same result as in the case with the CI method.

\subsection{Lagrangian of a particle in a constant strong magnetic field}

We consider the simple problem of a non-relativistic particle of charge $q$ and mass $m$, in a constant magnetic field $\vec{B}_{0}$ pointing in the $z$ direction. The Lagrangian is

$$
L=\frac{1}{2} m v^{2}+q \vec{A}(x, y) \cdot \vec{v}-q V(x, y)
$$


where $\vec{A}(x, y)$ is the vector potential in the Coulomb gauge $\vec{A}=\frac{1}{2} \vec{r} \wedge \overrightarrow{B_{0}}$ and $V(x, y)$ the scalar potential. In the limit of the strong magnetic field the mass term can be neglected and the Lagrangian is approximated as:

$$
L=\frac{q B_{0}}{2}(x \dot{y}-y \dot{x})-q V(x, y)
$$

In order to simplify the mathematics we consider the case $V(x, y)=\frac{1}{2} k\left(x^{2}+y^{2}\right)$, thus:

$$
L=\eta(x \dot{y}-y \dot{x})-\frac{1}{2} \xi\left(x^{2}+y^{2}\right)
$$

with $\eta=\frac{q B_{0}}{2}$ and $\xi=q k$.

\subsubsection{CI method}

The Euler-Lagrange equations are

$$
-2 \eta \dot{y}+\xi x=0 \quad ; \quad 2 \eta \dot{x}+\xi y=0
$$

or

The solutions are

$$
\ddot{x}+\frac{\xi^{2}}{4 \eta^{2}} x=0 \quad ; \quad \ddot{y}+\frac{\xi^{2}}{4 \eta^{2}} y=0
$$

$$
\begin{aligned}
x & =a \cos (\omega t)+b \sin (\omega t) \\
y & =-b \cos (\omega t)+a \sin (\omega t) \\
p_{x} & =\eta(b \cos (\omega t)-a \sin (\omega t)) \\
p_{y} & =\eta(a \cos (\omega t)+b \sin (\omega t))
\end{aligned}
$$

with $\omega=\frac{\xi}{2 \eta}$. The Hamiltonian of this system expressed in terms of the constants of integration is

$$
H=\frac{\xi}{2}\left(a^{2}+b^{2}\right)
$$

and the Hamilton equations $\dot{x}=\{x, H\}$ and $\dot{y}=\{y, H\}$ give the equalities

$$
\begin{aligned}
-a \omega \sin (\omega t)+b \omega \cos (\omega t) & =\{a, b\} b \xi \cos (\omega t)+\{b, a\} a \xi \sin (\omega t) \\
b \omega \sin (\omega t)+a \omega \cos (\omega t) & =-\{b, a\} a \xi \cos (\omega t)+\{a, b\} b \xi \sin (\omega t)
\end{aligned}
$$

from which we easily read off the bracket between the constants of integration $\{a, b\}=\frac{\omega}{\xi}$. From it we deduce the brackets between the dynamical variables

$$
\begin{aligned}
\{x, y\} & =-\frac{1}{q B_{0}} \\
\left\{x, p_{x}\right\} & =\left\{y, p_{y}\right\}=\frac{1}{2} \\
\left\{p_{x}, p_{y}\right\} & =\frac{1}{4} q B_{0}
\end{aligned}
$$




\subsubsection{Dirac approach}

We deduce from the same Lagrangian the following momenta $p_{x}=-\frac{q B_{0}}{2} y, p_{y}=\frac{q B_{0}}{2} x$ and the canonical Hamiltonian $H_{c}=\frac{\xi}{2}\left(x^{2}+y^{2}\right)$. We therefore have two primary constraints

$$
\begin{aligned}
\phi_{1} & =p_{x}+\frac{q B_{0}}{2} y \approx 0 \\
\phi_{2} & =p_{y}-\frac{q B_{0}}{2} x \approx 0
\end{aligned}
$$

The total Hamiltonian with the constraints is $H_{T}=H_{c}+\lambda_{1} \phi_{1}+\lambda_{2} \phi_{2}$. The consistency conditions give $\lambda_{1} \approx-\frac{k}{B_{0}} y$ and $\lambda_{2} \approx \frac{k}{B_{0}} x$. We also see that these constraints are second class as $\left\{\phi_{1}, \phi_{2}\right\}_{P B}=$ $-\left\{\phi_{2}, \phi_{1}\right\}_{P B} \approx q B_{0} \neq 0$.

The matrix of constraints is therefore

$$
M=\left(\begin{array}{cc}
0 & \left\{\phi_{1}, \phi_{2}\right\}_{P B} \\
\left\{\phi_{2}, \phi_{1}\right\}_{P B} & 0
\end{array}\right)=q B_{0}\left(\begin{array}{cc}
0 & 1 \\
-1 & 0
\end{array}\right)
$$

From this matrix we get the Dirac brackets of the system:

$$
\begin{aligned}
\{x, y\}_{D} & =-\frac{1}{q B_{0}} \\
\left\{x, p_{x}\right\}_{D} & =\left\{y, p_{y}\right\}_{D}=\frac{1}{2} \\
\left\{p_{x}, p_{y}\right\}_{D} & =\frac{1}{4} q B_{0}
\end{aligned}
$$

which is, as expected, are identical to some obtained with the CI method.

\subsubsection{Faddeev-Jackiw approach}

In this case the F-J Lagrangian is the same

$$
L_{F J}=\eta(x \dot{y}-y \dot{x})-\frac{1}{2} \xi\left(x^{2}+y^{2}\right)
$$

with $p_{x}=-\eta y$ and $p_{y}=\eta x$, the Euler-Lagrange equations are then

$$
\begin{aligned}
\eta \dot{y} & =-\eta \dot{y}+\xi x \\
-\eta \dot{x} & =\eta \dot{x}+\xi y
\end{aligned}
$$

or in matrix form

$$
2 \eta \underbrace{\left(\begin{array}{cc}
0 & 1 \\
-1 & 0
\end{array}\right)}_{f}\left(\begin{array}{l}
\dot{x} \\
\dot{y}
\end{array}\right)=\left(\begin{array}{l}
\xi x \\
\xi y
\end{array}\right)
$$

The inverse matrix is

$$
f^{-1}=\frac{1}{2 \eta}\left(\begin{array}{cc}
0 & -1 \\
1 & 0
\end{array}\right)=\left(\begin{array}{ll}
\{x, x\} & \{x, y\} \\
\{y, x\} & \{y, y\}
\end{array}\right)
$$

and we again find the same brackets as with the two other approaches. 


\subsection{Gauge invariant Lagrangian}

Consider now a system described by the Lagrangian

$$
L=\frac{1}{2}(y \dot{x}+x \dot{y})^{2} .
$$

\subsubsection{CI method}

The general solution of the equations of motion is obtained by introducing $Q=x y$, and consequently $\dot{Q}=y \dot{x}+x \dot{y}$. Then we have $\ddot{Q}=0$ leading to the following results

$$
\begin{aligned}
x(t) & =(a t+b) \varepsilon(t) \quad ; \quad y(t)=\frac{1}{\varepsilon(t)} \\
p_{x}(t) & =\frac{a}{\varepsilon(t)} ; \quad p_{y}(t)=\left(a^{2} t+a b\right) \varepsilon(t),
\end{aligned}
$$

where $\varepsilon(t)$ is an arbitrary function of time. To determine the infinitesimal gauge transformation that leaves the Lagrangian invariant we consider an infinitesimal variation $\delta \varepsilon$. This leads to $\delta x=$ $(a t+b) \delta \varepsilon$ and $\delta y=-\frac{\delta \varepsilon}{\varepsilon(t)^{2}}$ which can be written $\delta x=x y \delta \varepsilon$ and $\delta y=-y^{2} \delta \varepsilon$.

Fixing the gauge with the condition $y-1=0 \Rightarrow \varepsilon(t)=1$, the solution becomes

$$
\begin{aligned}
x(t) & =a t+b \quad ; \quad y(t)=1 \\
p_{x}(t) & =a \quad ; \quad p_{y}(t)=a^{2} t+a b .
\end{aligned}
$$

Replacing this solution in $H=\dot{x} p_{x}+\dot{y} p_{y}-L$, we obtain

$$
H=\frac{1}{2} a^{2}
$$

and using Hamilton equation $\frac{d x}{d t}=\{x, H\}$ in order to have directly the bracket

$$
\{a, b\}=-1
$$

From this bracket among the constants, we determine the fundamental brackets :

$$
\begin{aligned}
\{x, y\} & =\left\{y, p_{x}\right\}=\left\{y, p_{y}\right\}=0 \\
\left\{x, p_{x}\right\} & =1 \\
\left\{x, p_{y}\right\} & =x \\
\left\{p_{x}, p_{y}\right\} & =-p_{x} .
\end{aligned}
$$

\subsubsection{Dirac approach}

From the Lagrangian we can deduce the momenta $p_{x}=y(y \dot{x}+x \dot{y})$ and $p_{y}=x(y \dot{x}+x \dot{y})$. It is clear that we have a primary constraint $\phi=x p_{x}-y p_{y} \approx 0$. The canonical Hamiltonian is $H_{c}=\frac{1}{2} \frac{p_{x}^{2}}{y^{2}}$, and the total Hamiltonian is defined as $H_{T}=H_{c}+\lambda \phi$. The constraint is here first class because it is the only constraint. In order to define the Dirac brackets, it is known that one must add an additional 
condition fixing the gauge. Here we choose the same as previously $\zeta=y-1=0$. Therefore the matrix of constraints is

$$
M=\left(\begin{array}{ll}
\{\phi, \phi\}_{P B} & \{\phi, \zeta\}_{P B} \\
\{\zeta, \phi\}_{P B} & \{\zeta, \zeta\}_{P B}
\end{array}\right)=\left(\begin{array}{ll}
0 & y \\
-y & 0
\end{array}\right)=\left(\begin{array}{ll}
0 & 1 \\
-1 & 0
\end{array}\right) .
$$

Now we can calculate the Dirac brackets among the dynamical variables

$$
\begin{aligned}
\{x, y\}_{D} & =\{x, y\}_{P B}-\{x, \phi\}_{P B}\{\phi, \zeta\}_{P B}^{-1}\{\zeta, y\}_{P B}-\{x, \zeta\}_{P B}\{\zeta, \phi\}_{P B}^{-1}\{\phi, y\}_{P B}=0 \\
\left\{x, p_{x}\right\}_{D} & =\left\{x, p_{x}\right\}_{P B}-\{x, \phi\}_{P B}\{\phi, \zeta\}_{P B}^{-1}\left\{\zeta, p_{x}\right\}_{P B}-\{x, \zeta\}_{P B}\{\zeta, \phi\}_{P B}^{-1}\left\{\phi, p_{x}\right\}_{P B}=1 \\
\left\{x, p_{y}\right\}_{D} & =\left\{x, p_{y}\right\}_{P B}-\{x, \phi\}_{P B}\{\phi, \zeta\}_{P B}^{-1}\left\{\zeta, p_{y}\right\}_{P B}-\{x, \zeta\}_{P B}\{\zeta, \phi\}_{P B}^{-1}\left\{\phi, p_{y}\right\}_{P B}=x \\
\left\{y, p_{x}\right\}_{D} & =\left\{y, p_{x}\right\}_{P B}-\{y, \phi\}_{P B}\{\phi, \zeta\}_{P B}^{-1}\left\{\zeta, p_{x}\right\}_{P B}-\{y, \zeta\}_{P B}\{\zeta, \phi\}_{P B}^{-1}\left\{\phi, p_{x}\right\}_{P B}=0 \\
\left\{y, p_{y}\right\}_{D} & =\left\{y, p_{y}\right\}_{P B}-\{y, \phi\}_{P B}\{\phi, \zeta\}_{P B}^{-1}\left\{\zeta, p_{y}\right\}_{P B}-\{y, \zeta\}_{P B}\{\zeta, \phi\}_{P B}^{-1}\left\{\phi, p_{y}\right\}_{P B}=0 \\
\left\{p_{x}, p_{y}\right\}_{D} & =\left\{p_{x}, p_{y}\right\}_{P B}-\left\{p_{x}, \phi\right\}_{P B}\{\phi, \zeta\}_{P B}^{-1}\left\{\zeta, p_{y}\right\}-\left\{p_{x}, \zeta\right\}_{P B}\{\zeta, \phi\}_{P B}^{-1}\left\{\phi, p_{y}\right\}_{P B}=-p_{x} .
\end{aligned}
$$

These brackets are identical to those obtained with the CI method.

\subsubsection{Faddeev-Jackiw approach}

Using the primary constraint $x p_{x}-y p_{y}=0$, we define the FJ Lagrangian as $L_{F J}=\dot{x} p_{x}+\dot{y} p_{y}-H_{c}$ which reads

$$
L_{F J}=p_{x} \dot{x}+\frac{x p_{x}}{y} \dot{y}-\frac{1}{2} \frac{p_{x}^{2}}{y^{2}}
$$

The independent variables of the system are $x, y, z \equiv p_{x}$, then

$$
L_{F J}=z \dot{x}+\frac{x z}{y} \dot{y}-\frac{1}{2} \frac{z^{2}}{y^{2}}
$$

The Euler-Lagrange equations are

$$
\begin{aligned}
-\dot{z} & =-\frac{z}{y} \dot{y} \\
-\frac{\dot{x} z}{y}+\frac{x z}{y^{2}} \dot{y}-\frac{x \dot{z}}{y} & =\frac{x z}{y^{2}} \dot{y}-\frac{z^{2}}{y^{3}} \\
0 & =-\dot{x}-\frac{x}{y} \dot{y}+\frac{z}{y^{2}}
\end{aligned}
$$

which can also be written in the matrix form

$$
\underbrace{\left(\begin{array}{ccc}
0 & \frac{z}{y} & -1 \\
-\frac{z}{y} & 0 & -\frac{x}{y} \\
1 & \frac{x}{y} & 0
\end{array}\right)}_{f}\left(\begin{array}{l}
\dot{x} \\
\dot{y} \\
\dot{z}
\end{array}\right)=\left(\begin{array}{c}
0 \\
-\frac{z^{2}}{y^{3}} \\
\frac{z}{y^{2}}
\end{array}\right) .
$$

The matrix $f$ is antisymmetric and singular as $\operatorname{det}(f)=0$. The single zero mode is $\left(\begin{array}{ccc}-\frac{x}{z} & \frac{y}{z} & 1\end{array}\right)$ but it does not help as the zero mode equation does not lead to a secondary constraint equation. 
The matrix $f$ is therefore still singular and we are in the presence of a gauge symmetry. We choose the same gauge condition $y=1$ and add the term $\dot{\lambda}(y-1)$ to the $L_{F J}$ Lagrangian, i.e.;

$$
L_{F J}=z \dot{x}+\frac{x z}{y} \dot{y}-\frac{1}{2} \frac{z^{2}}{y^{2}}+\dot{\lambda}(y-1) .
$$

The Euler-Lagrange equations are now

$$
\begin{aligned}
-\dot{z} & =-\frac{z}{y} \dot{y} \\
-\frac{\dot{x} z}{y}+\frac{x z}{y^{2}} \dot{y}-\frac{x \dot{z}}{y} & =\frac{x z}{y^{2}} \dot{y}-\frac{z^{2}}{y^{3}}-\dot{\lambda} \\
0 & =-\dot{x}-\frac{x}{y} \dot{y}+\frac{z}{y^{2}} \\
-\dot{y} & =0
\end{aligned}
$$

or in the matrix form

$$
\underbrace{\left(\begin{array}{cccc}
0 & \frac{z}{y} & -1 & 0 \\
-\frac{z}{y} & 0 & -\frac{x}{y} & 1 \\
1 & \frac{x}{y} & 0 & 0 \\
0 & -1 & 0 & 0
\end{array}\right)}_{f}\left(\begin{array}{c}
\dot{x} \\
\dot{y} \\
\dot{z} \\
\dot{\lambda}
\end{array}\right)=\left(\begin{array}{c}
0 \\
-\frac{z^{2}}{y^{3}} \\
\frac{z}{y^{2}} \\
0
\end{array}\right)
$$

The matrix $f$ is now antisymmetric and invertible because $\operatorname{det}(f)=1 \neq 0$. The inverse matrix $f^{-1}$ is given by

$$
f^{-1}=\left(\begin{array}{cccc}
0 & 0 & 1 & \frac{x}{y} \\
0 & 0 & 0 & -1 \\
-1 & 0 & 0 & -\frac{z}{y} \\
-\frac{x}{y} & 1 & \frac{z}{y} & 0
\end{array}\right)=\left(\begin{array}{cccc}
\{x, x\} & \{x, y\} & \{x, z\} & \{x, \lambda\} \\
\{y, x\} & \{y, y\} & \{y, z\} & \{y, \lambda\} \\
\{z, x\} & \{z, y\} & \{z, z\} & \{z, \lambda\} \\
\{\lambda, x\} & \{\lambda, y\} & \{\lambda, z\} & \{\lambda, \lambda\}
\end{array}\right)
$$

and we find again

$$
\begin{aligned}
& \{x, y\}=\{y, z\}=0 \\
& \{x, z\}=1
\end{aligned}
$$

To obtain the brackets with $p_{y}$ one uses the primary constraint $p_{y}=\frac{x p_{x}}{y}$ and put $y=1$ at the end of the computation of the brackets.

We can see on this particular example that the Dirac and Faddeev-Jackiw approaches are closer to each other than the CI method.

\section{$5 \quad$ Hojman-Urrutia Lagrangian}

There are at least two physical motivations for the study of the theory associated to this Lagrangian that we would like to point out briefly. The first goes back to the publication of Hojman and Urrutia [9] where they built a Lagrangian from two systems of second order differential equations for which 
in principle no second-order Lagrangian exists (following the well known Douglas classification [10]). The second motivation goes back to the work of Feynman, reported by Dyson [1] who derived the first group of Maxwell equations from the classical equations of motion and imposed brackets among coordinates and velocities. This work has been extended to the two groups of Maxwell equations [12. Although with this "Feynman brackets" approach, there is no Lagrangian or Hamiltonian structure, Hojman and Shepley 13 showed by using a Helmholtz inverse method, that under certain conditions a Lagrangian can be associated to these Feynman brackets. In particular, in order to test their method they studied the following equations of motion

$$
\ddot{x}=-\dot{y} \quad \text { and } \quad \ddot{y}=-y
$$

which are not derivable from a Lagrangian. By rewriting the system in first order form as $\dot{x}=z$, $\dot{y}=\omega, \dot{z}=-\omega$ and $\dot{\omega}=-y$, they found the corresponding Lagrangian (by using integration constants in another context)

$$
L=(y+z) \dot{x}+\omega \dot{z}+\frac{1}{2}\left(\omega^{2}-2 y z-z^{2}\right) .
$$

This Lagrangian is singular and therefore this is a constrained system. This theory has been studied via the Dirac approach in [14] and by the Faddeev-Jackiw approach by [15]. We do not reproduce their results here but we will recover them from the CI method which turns out to be much simpler.

\subsection{First case: $\omega$ not constant}

The Euler-Lagrange equations are

$$
\begin{array}{cc}
\dot{y}+\dot{z}=0 & \dot{x}=z \\
0=\dot{x}-z & \dot{y}=\omega \\
\dot{\omega}=\dot{x}-y-z & \Leftrightarrow \quad \dot{z}=-\omega \\
0=\dot{z}+\omega & \dot{\omega}=-y
\end{array}
$$

therefore $\ddot{y}=-y$ and $\ddot{x}=-y$ (starting equations of Hojman and Shepley [13]).

The general solution is

$$
\begin{aligned}
x & =a \cos (t)+b \sin (t)+c t+d \\
y & =-b \cos (t)+a \sin (t) \\
z & =-a \sin (t)+b \cos (t)+c \\
\omega & =a \cos (t)+b \sin (t)
\end{aligned}
$$

and the Hamiltonian is

$$
H=-\frac{1}{2}\left(a^{2}+b^{2}-c^{2}\right)
$$

From the Hamilton equation for $x$ we obtain the equality

$$
\begin{aligned}
-a \sin (t)+b \cos (t)+c= & (-\{a, b\} b+\{a, c\} c) \cos (t)+(\{a, b\} a+\{b, c\} c) \sin (t) \\
& +(\{b, c\} b+\{a, c\} a) t+(\{b, d\} b+\{a, d\} a-\{c, d\}) c
\end{aligned}
$$


In the same manner for $y$

$$
b \sin (t)+a \cos (t)=(-\{a, b\} a+\{b, c\} c) \cos (t)-(\{a, b\} b+\{a, c\} c) \sin (t)
$$

which gives directly by identification

$$
\begin{aligned}
& \{a, b\}=\{c, d\}=-1 \\
& \{a, c\}=\{a, d\}=\{b, c\}=\{b, d\}=0
\end{aligned}
$$

and finally

$$
\begin{aligned}
& \{x, y\}=\{z, \omega\}=1 \\
& \{y, \omega\}=-1 \\
& \{x, z\}=\{x, \omega\}=\{y, z\}=0
\end{aligned}
$$

Again, we obtain the same results as with the other methods. The reader will notice the efficiency of our approach.

\subsection{Second case: $\omega$ constant}

This case with $\omega=k$ constant studied also in [15 is interesting as it leads to a gauge theory [14 . In this case, the Euler-Lagrange equations are

$$
\begin{aligned}
\dot{z} & =0 \\
\dot{x}-z & =0 \\
\dot{x}-z-y & =0
\end{aligned}
$$

whose general solution is

$$
\begin{aligned}
& x=a t+b \\
& y=0 \\
& z=a
\end{aligned}
$$

The Hamiltonian is then

$$
H=\frac{1}{2}\left(k^{2}-2 y z-z^{2}\right)=\frac{1}{2} a^{2}
$$

and from the Hamilton equation $\dot{x}=\{x, H\}$ we obtain the equation $a=\left\{b, \frac{1}{2} a^{2}\right\}$ which gives directly the bracket among the constants

$$
\{a, b\}=-1
$$

From this we obtain the following brackets

$$
\begin{aligned}
& \{x, z\}=1 \\
& \{x, y\}=\{y, z\}=0
\end{aligned}
$$

as expected. 


\section{Application to the Dirac field}

Here we present a different perspective on the quantization of free fields based on the method we developed here. The idea is that, instead of applying the quantization rules (for bosons and fermions) based on the correspondence principle among the fields and their momenta [16, we simply assume the validity of the Heisenberg equation of motion of the field operators. Then we treat the creation and annihilation operators in the same manner as the constants of motion of the classical equations of motion. As an example we consider the Dirac field $\Psi$, with the Lagrangian density

$$
\mathcal{L}=i \bar{\Psi} \gamma^{\mu} \partial_{\mu} \Psi-m \bar{\Psi} \Psi
$$

where $\gamma^{\mu}$ are the Dirac matrices. The Euler-Lagrange equations are $i \gamma^{\mu} \partial_{\mu} \Psi-m \Psi=0$ and $i \bar{\Psi} \gamma^{\mu} \overleftarrow{\partial}_{\mu}$ $+m \bar{\Psi}=0$ whose general solution is

$$
\Psi=\int \sum_{s=1}^{2} d \mathbf{k}\left(f_{k}(x) b_{s}(\mathbf{k}) u_{s}(\mathbf{k})+f_{k}^{*}(x) d_{s}^{\dagger}(\mathbf{k}) v_{s}(\mathbf{k})\right)
$$

where $f_{k}(x)=\sqrt{\frac{m}{(2 \pi)^{3} k_{0}}} e^{-i k x}, k_{0}=\sqrt{\mathbf{k}^{2}+m^{2}}$ and $u_{s}(\mathbf{k})$ and $v_{s}(\mathbf{k})$ are the usual bispinors. The $b_{s}(\mathbf{k})$ and $d_{s}(\mathbf{k})$ are operators playing the role of the constants of motion in the classical case (before quantization). Then as previously we write the Hamiltonian in terms of these operators

$$
H=\int d \mathbf{k} k_{0} \sum_{s=1}^{2}\left(b_{s}^{\dagger}(\mathbf{k}) b_{s}(\mathbf{k})-d_{s}(\mathbf{k}) d_{s}^{\dagger}(\mathbf{k})\right)
$$

From the Heisenberg equation

$$
\dot{\Psi}=\frac{1}{i \hbar}[\Psi, H]
$$

We obtain the following equalities

$$
\begin{aligned}
& k_{0} b_{s}(\vec{k})=\int d \vec{k}^{\prime} k_{0}^{\prime} \sum_{s^{\prime}=1}^{2}\left[b_{s}(\vec{k}), N_{s^{\prime}}\left(\vec{k}^{\prime}\right)\right] \\
& k_{0} d_{s}^{\dagger}(\vec{k})=-\int d \vec{k}^{\prime} k_{0}^{\prime} \sum_{s^{\prime}=1}^{2}\left[d_{s}^{\dagger}(\vec{k}), N_{s^{\prime}}\left(\vec{k}^{\prime}\right)\right]
\end{aligned}
$$

with $N_{s}(\vec{k})=\left(b_{s}^{\dagger}(\mathbf{k}) b_{s}(\mathbf{k})-d_{s}(\mathbf{k}) d_{s}^{\dagger}(\mathbf{k})\right)$. From these equations we directly read off the following brackets

$$
\begin{gathered}
{\left[b_{s}(\vec{k}), N_{s^{\prime}}\left(\vec{k}^{\prime}\right)\right]=b_{s^{\prime}}\left(\vec{k}^{\prime}\right) \delta_{s s^{\prime}} \delta\left(\vec{k}-\vec{k}^{\prime}\right)} \\
{\left[d_{s}^{\dagger}(\vec{k}), N_{s^{\prime}}\left(\vec{k}^{\prime}\right)\right]=-d_{s^{\prime}}^{\dagger}\left(\vec{k}^{\prime}\right) \delta_{s s^{\prime}} \delta\left(\vec{k}-\vec{k}^{\prime}\right) .}
\end{gathered}
$$

For fermions, we express these commutators by means of anticommutators

$$
\begin{aligned}
{\left[b_{s}(\vec{k}), N_{s^{\prime}}\left(\vec{k}^{\prime}\right)\right]=} & -b_{s^{\prime}}^{\dagger}\left(\vec{k}^{\prime}\right)\left\{b_{s}(\vec{k}), b_{s^{\prime}}\left(\vec{k}^{\prime}\right)\right\}+\left\{b_{s}(\vec{k}), b_{s^{\prime}}^{\dagger}\left(\vec{k}^{\prime}\right)\right\} b_{s^{\prime}}\left(\vec{k}^{\prime}\right) \\
& +d_{s^{\prime}}\left(\vec{k}^{\prime}\right)\left\{b_{s}(\vec{k}), d_{s^{\prime}}^{\dagger}\left(\vec{k}^{\prime}\right)\right\}-\left\{b_{s}(\vec{k}), d_{s^{\prime}}\left(\vec{k}^{\prime}\right)\right\} d_{s^{\prime}}^{\dagger}\left(\vec{k}^{\prime}\right)
\end{aligned}
$$


and

$$
\begin{aligned}
{\left[d_{s}^{\dagger}(\vec{k}), N_{s^{\prime}}\left(\vec{k}^{\prime}\right)\right]=} & -b_{s^{\prime}}^{\dagger}\left(\vec{k}^{\prime}\right)\left\{d_{s}^{\dagger}(\vec{k}), b_{s^{\prime}}\left(\vec{k}^{\prime}\right)\right\}+\left\{d_{s}^{\dagger}(\vec{k}), b_{s^{\prime}}^{\dagger}\left(\vec{k}^{\prime}\right)\right\} b_{s^{\prime}}\left(\vec{k}^{\prime}\right) \\
& +d_{s^{\prime}}\left(\vec{k}^{\prime}\right)\left\{d_{s}^{\dagger}(\vec{k}), d_{s^{\prime}}^{\dagger}\left(\vec{k}^{\prime}\right)\right\}-\left\{d_{s}^{\dagger}(\vec{k}), d_{s^{\prime}}\left(\vec{k}^{\prime}\right)\right\} d_{s^{\prime}}^{\dagger}\left(\vec{k}^{\prime}\right)
\end{aligned}
$$

then by identification of Eqs. (14) and (16) we obtain the anticommutation rule

$$
\left\{b_{s}(\vec{k}), b_{s^{\prime}}^{\dagger}\left(\vec{k}^{\prime}\right)\right\}=\delta_{s s^{\prime}} \delta\left(\vec{k}-\vec{k}^{\prime}\right)
$$

In the same manner, the identification between Eqs. (15) and (18) gives

$$
\left\{d_{s^{\prime}}\left(\vec{k}^{\prime}\right), d_{s}^{\dagger}(\vec{k})\right\}=\delta_{s s^{\prime}} \delta\left(\vec{k}-\vec{k}^{\prime}\right)
$$

These rules of quantization are exactly identical to the results of the canonical quantization of the Dirac field. The same approach can be used for all quantum fields (Maxwell and Klein-Gordon).

\section{Conclusion}

The quantization of constrained systems is an old topic going back to Dirac and Bergmann. Their approach with the introduction of first and second class, primary and secondary constraints is essential but is very often difficult to apply. Faddeev and Jackiw proposed an alternative approach based on symplectic geometry and Darboux theorem which is very efficient and generally simpler than Dirac approach. In this paper, we developed another method that was applied on several examples and compared with the two other approaches. This method requires the knowledge of the general solutions of the equations of motion. This is for instance similar to the semiclassical quantization from the path integral approach. It is practical because it is very direct, easy and without special formalism. The novelty of this approach is based on the (simple) computation of the (Dirac) brackets among the integration constants (CI method) of the equations of motion. These constants are therefore considered as variables, a method reminiscent of the Hamilton-Jacobi calculus. We found that the CI method is consistent with the two others and we believe that it is also simpler in general. It is however based on the knowledge of the analytical solution, which is its weak point. When the classical analytical solution is not known, the method can still be applied by starting from the free systems because the solution is accessible, and then use the perturbation method to go further. Even in the others approaches, Dirac-Bergmann and Faddeev-Jackiw methods, one must find the free solution after quantization in order to use the perturbation development. In the near future we plan to extend our approach to non soluble and more complicated constrained systems like spinning particles in an electromagnetic field [17] or relativistic particles in non-commutative space-time [18. Beyond its technical aspect this method sheds light also on another point of view of the quantization process. Indeed, assuming only the validity of Hamilton (or Heisenberg) equations for the quantum fields, the quantization rules emerge naturally instead of postulating the commutation rules among the fields.

Acknowledgements. We thank M. M. Müller and Y. Grandati for stimulating discussions. Z. B. thanks A. H. Gharbi for stimulating discussions and for support. 


\section{Bibliography}

\section{References}

[1] P.A.M. Dirac, Lectures on Quantum Mechanics, Belfer Graduate School of Science (1964).

[2] J. L. Anderson and P. G. Bergmann, "Constraints in covariant field theories", Phys. Rev. 83 (1951) 1018.

[3] L. Faddeev and R. Jackiw, "Hamiltonian Reduction of Unconstraint and Constraint Systems" Phys. Rev. Lett. 60 (1988) 1692.

[4] L. Liao and Y. C. Huang, "Non-equivalence of Faddeev-Jackiw method and Dirac-Bergmann algorithm and the modification of Faddeev-Jackiw method for keeping the equivalence", Ann. Phys. 322 (2007) 2469.

[5] J. M. Souriau, "Structures des systèmes dynamiques", Paris, Dunod (1969); "Structure of Dynamical Systems, a Symplectic View of Physics", Birkäuser (1997).

[6] C. Duval and P. Horvathy, "The exotic Galilei group and the "Peierls substitution", Phys. Lett. B. 479 (2000) 284.

[7] l. Martina, "Dynamics with exotic symmetries", Jour. Phys. Conf. Ser. 343 (2012) 012072.

[8] J. F. Cariñena, M. F. Rañada, M. Santander, "A nonlinear deformation of the isotonic oscillator and the Smorodinski-Winternitz system : integrability and superintegrability, R and C Dynamics (DOI: 10.1070/RD2005v010n04ABEH000324).

[9] S. Hojman and L.F. Urrutia, "On the inverse problem of the calculus of variations", J. Math. Phys. 22 (1981) 1896.

[10] J. Douglas, "Solution of the inverse problem of the calculus of variations", Trans. Am. Math. Soc. 50 (1941) 71.

[11] F. Dyson, Am. J. Phys. 58, 209 (1990).

[12] A. Bérard, Y. Grandati and H. Mohrbach, "Dirac monopole with Feynman brackets", Phys. Lett. A. 254 (1999) 133; "Magnetic Monopole in the Feynman derivation of Maxwell equations", J. Math. Phys. 40 (1999) 3732.

[13] S. Hojman and L. C. Shepley, "No Lagrangian, no quantization !", J. Math. Phys. 32 (1991) 142 .

[14] U. Kulshreshtha " Hamiltonian formulation of a theory with constraints", J. Math. Phys. 33 (1991) 633; J. Math. Phys. 33 (1991) 4066.

[15] J. Barcelos-Neto and N. R. F. Braga, "Symplectic analysis of a Dirac constrained theory", J. Math.Phys. 35 (1994) 3497.

[16] C. Itzykson and J. B. Zuber, "Quantum Field Theory", McGraw-Hill (1980). 
[17] A. Bérard, S. Ghosh, Y. Grandati, H. Mohrbach, P. Pal, "Constrained dynamics of an anomalous $(g \neq 2)$ relativistic spinning particle in electromagnetic background", Eur. Phys. J. C, 71 (2011) 1770; P. A. Horvathy and M. S. Plyushchay, "Nonrelativistic anyons in external electromagnetic field", Nucl. Phys. B714 (2005) 269.

[18] S. Ghosh, "Deformed Special Relativity and Deformed Symmetries in a Canonical Framework" Phys. Rev. D 75 (2007) 105012; M. Chaichian, S. Ghosh, M. Langvik, A. Tureanu, "Dirac Quantization Condition for Monopole in Noncommutative Space-Time", Phys. Rev. D 79 (2009) 125029. 